\title{
A Grid of Remote Laboratory for Teaching Electronics
}

\author{
Andrea Bagnasco, Anna Marina Scapolla \\ DIBE - Department of Biophysical and Electronic Engineering \\ University of Genova \\ Via Opera Pia 11/a, 16145, Genova, Italy \\ Phone: +39-010-3532267, Fax: +39-010-3522175 \\ \{bagnasco,scapolla\}@dibe.unige.it
}

\begin{abstract}
Testing theories through practice is an important approach to teaching, especially in scientific and technical curricula. Often, lack of resources and logistics problems makes practice impossible. During these last years, several attempts to find an alternative to in-laboratory experiments have been proposed from many researchers. In this paper we present our approach to remote laboratories challenge. We outline the model we have created and the prototype we have implemented and validated. Finally, we propose a grid-oriented vision of our work that could be an effective solution to support experimental activity in e-learning efforts among a scattered community of users.
\end{abstract}

Keywords: Web-based Remote Laboratory, E-learning, Grid.

\section{Teaching electronics with remote labs}

Multimedia and network interactivity are leading to new forms of teaching and learning and new roles for students who act as participants and not only spectators of their own learning process. Testing theories through practice is an important approach to teaching, especially in scientific and technical curricula. Often, lack of resources, logistics problems, disabilities and hazardous or dangerous environment conditions make practice impossible. During these last years, riding the e-learning wave, several attempts to find an alternative to in-laboratory experiments have been done and literature in this field is quite large. Some examples are given in [1, 2, 3, 4, and 5]. Today's lab experiences can be based on simulations or, alternatively, the network can provide a remote access to personal computers that control real instrumentation and physical devices. These approaches lead to effective solution for widespread and continuous education, generally limited by time and space constraints, special education based on the use of expensive and rare equipment, geographically scattered, improving cooperation between researchers from different institutions.

A large amount of work has been done in this field from different groups of researchers, who presented various solutions. In the next section we will illustrate a model to share laboratories in Internet called ISILab (pronounced "easy-lab") [6].

\section{The ISILab approach}

ISILab is modelled as a distributed environment (see Figure 1) consisting of a main Virtual Laboratory Server (VLS), one or more Real Laboratory Servers (RLS) and user/client stations. Internet links all these components. 


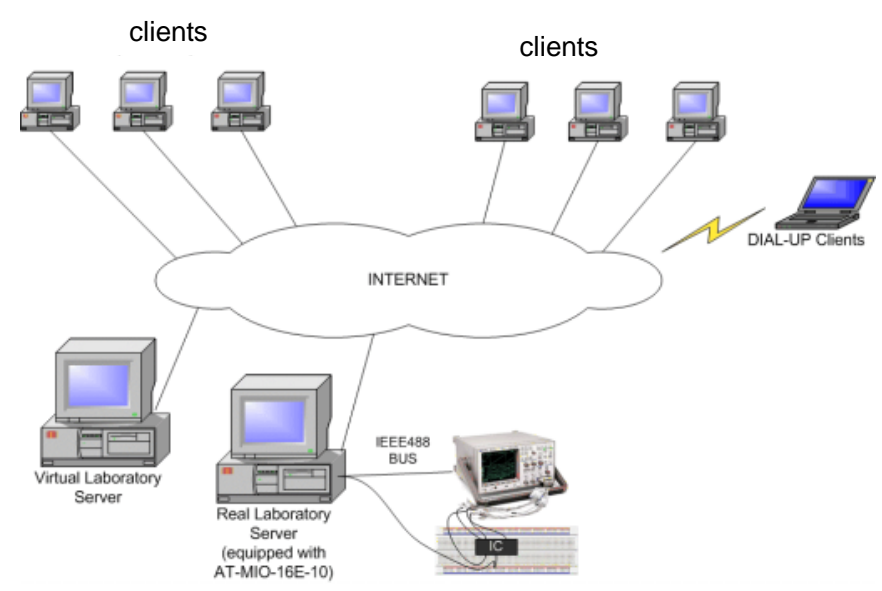

FIGURE 1: ISILab Architecture

RLSs can be spread on wide geographic area and control real experiments, managing sets of heterogeneous resources (i.e. measurement instrumentation). No limit exists to the location of the laboratories and the only requirement is an Internet connection between client and servers controlling resources. Users do not require any particular hardware or software and can carry out experiments through the network. They work transparently to the real location of the device under test, in a multi-user concurrent way.

The environment allows users to carry out experiments in two different ways, guided mode and independent mode. In guided mode there is a privileged user who is the only one able to modify interactively the operational conditions, acting on the instrumentation controls, and the other users are only able to see the response of the system on their computer screens. This mode can be very effective in the context of distance education as teachers can show real laboratory experiments via Internet. When an experiment is carried out in independent mode all users are able to interact with actual instruments in parallel fashion and see only the results related to their own commands. The coherency of each experimental session is guaranteed by the RLS that take cares of separating different users' data spaces. Also the RLS provides to execute measurement applying these setting to the actual instruments in batch mode.

Another noticeable feature of ISILab is its modular and scaling structure, achieved through the decomposition of the experiment entity as a set of separate, independent, and reusable components, that can be assembled in order to create new and different experiences. Many developers can contribute to increase the number of experiments.

A prototype of the remote laboratory has been presented and validated. Students, remotely accessing the laboratory, are introduced to a set of experiments/lessons leading to gain knowledge of electronic instruments, measurement procedures and circuits under test. Each experiment is presented as a lecture, characterized a well-defined objective and a set of exercises that can be carried out via the web. A set of hints, tips and instrument handbooks are available on-line and provide a continuous support to conduct experiments.

Figure 2 is a snapshot of the prototype. It is worth noting that it is possible to have both simplified and very realistic virtual panels, in order to hit different didactical target. 


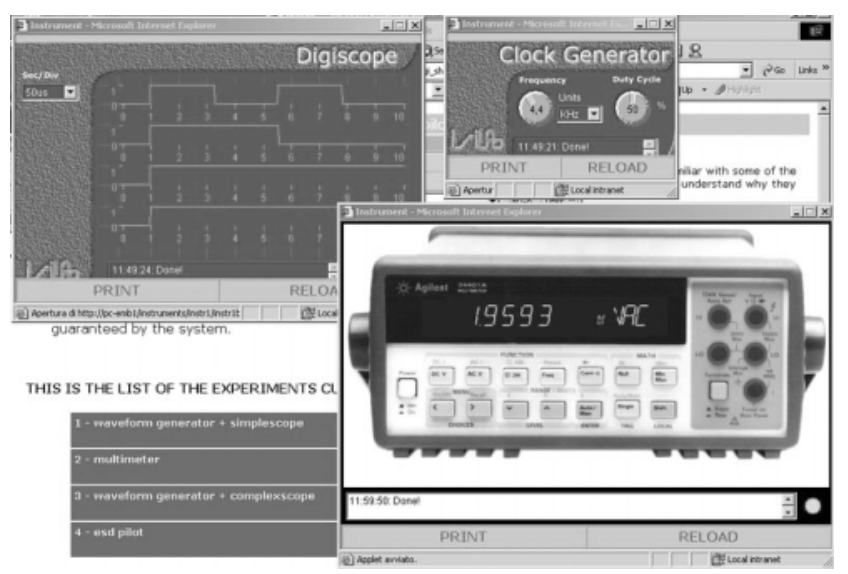

FIGURE 2: The learning environment

The entire server side of ISILab, both the RLS and the VLS, has been developed using LabVIEW [7].

The use of HTML and Java on the client side renders the system accessible by the simple use of a common web browser. The XML description of the entire data set associated to the environment assures maximum portability.

\section{Validation test}

A validation test has been carried out according to the work plan of the NetPro project [8], a EU Leonardo Da Vinci Programme initiative. ISILab has been applied to the teaching of delays in digital circuits, part of a course on digital electronics. Both RLS and VLS are hosted on an entry level standard PC (Pentium II 350 MHz, 192 MB RAM, Windows 2000) equipped with:

- A multifunction I/O card (National Instruments AT-MIO-16E-10)

- An IEEE488 interface card (National Instrument AT-GPIB) connecting a digital oscilloscope 54645D from HP/Agilent featuring 2 analogs and 16 digital channels.

The circuits to test have been built on an ordinary breadboard. The input of the circuit, for both the experiments, was taken from one of the two clock signals available from the $1 / O$ card. The output pins of the circuit have been connected to the analogue channels of oscilloscope for experiment 1 and to the digital ones for experiment 2.

Three specific user interfaces have been built to control the features of:

- clock generator,

- analogic oscilloscope,

- digital oscilloscope.

Students accessed the laboratory from different sites connected via the Internet and over dial-up lines. In order to limit the load of the ISILab server and to better monitor its behaviour, the laboratory session was executed in controlled way, limiting the number of clients able to connect to RLS. All the students worked in concurrent mode. We have established turns, in order to have a maximum of thirteen concurrent accesses each time. The number of sessions (one hour each) was 16 . In this way we have been able to guarantee a response time from the system of about one second. The analysis of the log files shows that the average number of actions for each experimental session is about seven per minute, while the response time of the system is less than 10 milliseconds, proving the feasibility of the system.

\section{A grid vision of isilab}

ISILab is characterised by a modular design to guarantee the possibility to extend the environment by including a wide range of equipment, eventually scattered. We are defining 
procedures to scale up laboratory experiences, using XML and related technologies to describe resources. Furthermore the system includes facilities to support the web management of the environment (teacher support, instruments/experiences add on, users administration, activity logs). The target is the cross-institutional integration of experimental resources: an environment where actors of a virtual organization [9] cooperate sharing geographically distributed educational resources that become part of a learning grid infrastructure. In this vision, referring the terminology adopted in [10], the VLS acts as a super scheduler. Users discover resource through the VLS. The VLS has responsibility for authorizing, filtering and selecting the system where the job must be run. If more than one RLS hosts the same experiment, the VLS has to consider the quality of service available from each candidate RLS. It is expressed in terms of the number of connected users and the available bandwidth. Once the most appropriate RLS has been chosen, the user starts his/her job. She/he can, eventually, exploit other features from the VLS to accomplish complementary tasks such as to seeking advice from documentation or annotating results. For performance or reliability reasons, the VLS can be duplicated. From the user's point of view, the VLS acts as a provider of learning resources, and our target is the alignment of the environment to the standard framework proposed for accessing web services [11]. It's worth noting that we have implemented the entire server side, thus both RLS and VLS, using LabVIEW programming language that per se guarantees multi-platform capability. In fact, programs developed with LabVIEW (the so-called $\mathrm{VI}$ ) are able to run, without modifications, on Microsoft Windows, Linux, Sun Solaris ${ }^{\mathrm{TM}}$ and Mac OSs. The use of HTML and Java on client side renders the system accessible by the simple use of a common web browser. The $\mathrm{XML}$ description of the entire data set associated to the environment assures the maximum portability.

To better understand possible matching between ISILab and the Grid model it can be useful to map the components of the ISILab paradigm on the Grid layer diagram [12] as illustrated in Figure 3. At the lowest level of this model, called fabric layer, there are the physical devices or resources that Grid users want to share and access. They can include computers, storage systems, catalogues, networks, and various forms of sensors and devices. Above of it, there is the connectivity layer, which contains the core communication and authentication protocols required for Grid-specific network transactions. One step over, the resource layer provides protocols that exploit communication enabling the secure initiation, monitoring, and control of resource-sharing operations. Then, the collective layer contains protocols, and services that implement interactions across collections of resources. At the top of the Grid system there are the user applications that control the components in any layer below. From the perspective of ISILab at the fabric layer there is the experiment, which is composed by actual instruments and the RLS's software modules that locally control them in order to execute concurrent measurement tasks. VLS is the module that offers collective management services. It brokers resources for the user, acting as a client for the RLS and as a server for the user application. At the user application layer there are the interfaces that let user to control the experiment execution.

\begin{tabular}{|c|c|c|}
\hline $\begin{array}{l}\text { USER } \\
\text { APPLICATIONS }\end{array}$ & Tools and applications & $\begin{array}{l}\text { Instrument's } \\
\text { GUI }\end{array}$ \\
\hline $\begin{array}{l}\text { COLLECTIVE } \\
\text { SERVICES }\end{array}$ & $\begin{array}{l}\text { Directory brokering, } \\
\text { diagnostic and } \\
\text { monitoring }\end{array}$ & VLS \\
\hline $\begin{array}{l}\text { RESOURCE AND } \\
\text { CONNECTIVITY } \\
\text { PROTOCOLS }\end{array}$ & $\begin{array}{l}\text { Secure access } \\
\text { to resources } \\
\text { and services }\end{array}$ & $\begin{array}{l}\text { Specific } \\
\text { TCP-based } \\
\text { protocols }\end{array}$ \\
\hline \multirow{2}{*}{ FABRIC } & \multirow[t]{2}{*}{ Diverse resources } & RLS \\
\hline & & $\begin{array}{l}\text { Actual } \\
\text { instrument }\end{array}$ \\
\hline
\end{tabular}

FIGURE 3: Mapping ISILab modules on a Grid model 
At the moment the schema is developed using proprietary protocols and APIs. We are now investigating the possibility of standardizing our approach integrating the system with the services offered from the Globus [13] framework. We are also studying the opportunities offered by Microsoft .NET technology.

\section{CONCLUSIONS}

In this paper we have presented our approach to remote laboratories challenge. Furthermore we have outlined the model we have created and the prototype we have implemented and validated. We have proposed a grid-oriented vision of our current work. We think that our approach can be an effective solution to support experimental activity in e-learning efforts among a scattered community of experiment providers and users.

\section{REFERENCES}

1 Grima Palop, J. M., \& Andres Teruel J. M. (Feb. 2000) "Virtual Workbench for Electronic Instrumentation Teaching", IEEE Transactions on Education, Vol. 43, No. 1.

2 Shen, H. et al. (August 1999) "Conducting Laboratory Experiments over the Internet", IEEE Transactions on Education, Vol. 42, No. 3.

3 Ko, C. C., et al. (April 2000) A Large Scale Web Based virtual Oscilloscope Laboratory Experiment, Engineering Science and Educational Journal.

4 Arpaia, P., Baccigalupi, A., Cennamo,F.,\& Daponte, P. (October 2000) A Measurement Laboratory on Geographic Network for Remote Test Experiments, IEEE Transactions On Instrumentation and Measurement, Vol. 49, No. 5.

5 Chung Ko, Chi et al. (August, 2001) A Web-Based Virtual Laboratory on a Frequency Modulation Experiment, IEEE Transactions on Systems, Man, and Cybernetics, Vol. 31, No. 3.

6 Bagnasco, Chirico, Scapolla. (2002) XML Technologies to Design Didactical Distributed Measurement Laboratories, IMTC.

$7 \mathrm{http}: / /$ www.ni.com/labview

8 The NetPro Project, http://netpro.evitech.fi/

9 Foster, Kesselman and Tuecke. (2001) The Anatomy of the Grid: Enabling Scalable Virtual Organizations, International J. Supercomputer Applications, 15(3).

10 Schopf. J. M. Ten Actions When Superscheduling. Available on-line at

http://www.gridforum.org/Documents/GFD/GFD-I.4.pdf

$11 \mathrm{http}: / / \mathrm{www} . \mathrm{w} 3.0 \mathrm{rg} / 2002 / \mathrm{ws} /$

12 Foster, I. (February 2002) The Grid: A New Infrastructure for 21st Century Science, Physics Today,.

$13 \mathrm{http}: / /$ www.globus.org 